\title{
Functional Insights into Recombinant TROSPA Protein from Ixodes ricinus
}

\author{
Marek Figlerowicz ${ }^{1,3 *}$, Anna Urbanowicz ${ }^{1}$, Dominik Lewandowski ${ }^{1}$, Jadwiga Jodynis-Liebert ${ }^{2}$, \\ Czeslaw Sadowski ${ }^{2}$
}

1 Institute of Bioorganic Chemistry, Polish Academy of Sciences, Poznan, Poland, 2 Department of Toxicology, Poznan University of Medical Sciences, Poznan, Poland, $\mathbf{3}$ Institute of Computing Science, Poznan University of Technology, Poznan, Poland

\begin{abstract}
Lyme disease (also called borreliosis) is a prevalent chronic disease transmitted by ticks and caused by Borrelia burgdorferi s. I. spirochete. At least one tick protein, namely TROSPA from I. scapularis, commonly occurring in the USA, was shown to be required for colonization of the vector by bacteria. Located in the tick gut, TROSPA interacts with the spirochete outer surface protein $\mathrm{A}(\mathrm{OspA})$ and initiates the tick colonization. Ixodes ricinus is a primary vector involved in B. burgdorferi s. I. transmission in most European countries. In this study, we characterized the capacities of recombinant TROSPA protein from I. ricinus to interact with OspA from different Borrelia species and to induce an immune response in animals. We also showed that the N-terminal part of TROSPA (a putative transmembrane domain) is not involved in the interaction with OspA and that reduction of the total negative charge on the TROSPA protein impaired TROSPA-OspA binding. In general, the data presented in this paper indicate that recombinant TROSPA protein retains the capacity to form a complex with OspA and induces a significant level of IgG in orally immunized rats. Thus, l. ricinus TROSPA may be considered a good candidate component for an animal vaccine against Borrelia.
\end{abstract}

Citation: Figlerowicz M, Urbanowicz A, Lewandowski D, Jodynis-Liebert J, Sadowski C (2013) Functional Insights into Recombinant TROSPA Protein from Ixodes ricinus. PLoS ONE 8(10): e76848. doi:10.1371/journal.pone.0076848

Editor: Brian Stevenson, University of Kentucky College of Medicine, United States of America

Received June 18, 2013; Accepted August 26, 2013; Published October 18, 2013

Copyright: (C) 2013 Figlerouicz et al. This is an open-access article distributed under the terms of the Creative Commons Attribution License, which permits unrestricted use, distribution, and reproduction in any medium, provided the original author and source are credited.

Funding: The research described in this work was co-funded by European Union within European Regional Development Fund through MPD programme and by the Polish Government through grant N N302 041536. The publication costs were covered by the European Regional Development Fund under the Operational Programme Innovative Economy - Grants for innovation. The funders had no role in study design, data collection and analysis, decision to publish, or preparation of the manuscript.

Competing Interests: The authors have declared that no competing interests exist.

*E-mail: Marek.Figlerowicz@ibch.poznan.pl

\section{Introduction}

Hard ticks of the genus Ixodes are ectoparasites of vertebrates. Ticks feed on animal blood, and consequently, they may function as vectors for numerous pathogenic microorganisms of vertebrates. Among other microbes, ticks transmit Borrelia burgdorferi sensu lato (s. l.), the causative agent of Lyme disease, also called borreliosis. $B$. burgdorferi s. $l$. is a phylogenetic group clustering numerous Borrelia species including the most common B. burgdorferi sensu stricto (s. s.), B. afzelii and B. garinii [1]. These spirochetes are widely spread all over the temperate zone of the northern hemisphere $[2,3,4,5,6,7]$. Ixodes ricinus is a primary vector for $B$. burgdorferi $s$. $l$. in most European countries, while Ixodes scapularis spreads mainly $B$. burgdorferi s. s. - a bacterial species typical for endemic areas of the USA [1]. The percentage of Borrelia-infected I. ricinus ticks in Europe ranges from a few to several dozen, depending on the region $[4,7,8]$. In the USA, the prevalence of Borrelia in ticks reaches up to sixty percent $[3,9,10]$. Borreliosis in humans is a serious chronic disease affecting multiple organ systems including the nervous system, cardiovascular system, muscles and joints. However, humans are only accidental hosts for Borrelia spirochetes. In a natural environment, the reservoir of Borrelia are small, wild vertebrates, mainly rodents [1].

Borrelia from the blood of an infected animal enters the tick during feeding. At least one tick protein, namely TROSPA from $I$. scapularis, was proven to be crucial for the colonization of the vector by bacteria. Located in the tick gut, TROSPA interacts with the spirochete outer surface protein A (OspA) [11]. The interaction between these two proteins allows the spirochetes to associate with the gut tissue, which is the first step of tick colonization. The data suggest that TROSPA is subjected to extensive post-translational modifications in vivo. Most likely glycosylated, $55 \mathrm{kDa}$ TROSPA protein was found in I. scapularis. A $16 \mathrm{kDa}$ protein was formed when the TROSPA encoding gene from I. scapularis was expressed in a bacterial system. Although this protein lacked post-translational modifications, it was capable of binding to OspA in a similar manner as to native glycosylated TROSPA [11]. Recently, the TROSPA homolog from I. persulcatus has also been identified [12]. This protein was predicted to possess a putative transmembrane helix at the $\mathrm{N}$-terminus $[11,12]$. Although the interaction between Ixodes TROSPA and spirochete OspA is indispensable for the circulation of Borrelia between the vector and the host, little is known about the nature of this interaction. In addition, the majority of the data collected so far have been obtained using TROSPA from I. scapularis, which is typical for the USA but not for Europe.

To date, approaches to prevent Borrelia infections have been focused on bacterial outer surface proteins [13,14,15]. For example, the vaccinations with OspA conferred a high level of resistance to borreliosis in the USA [13,14]. Unfortunately, some disadvantages of the outer surface proteins-based vaccine were 
reported. These included high variability of antigens in the individual Borrelia strains (causing OspA-based vaccine to confer the immunity only to one particular strain of Borrelia), antigenic differences among spirochetes occurring in different parts of the world (especially in Europe) and adverse side effects (e.g., arthritis) $[1,13,15]$. As a result, an idea was proposed to develop a tick protein-based vaccine against borreliosis. In addition, the possibility of a Borrelia reservoir vaccination has been considered [15]. The involvement of Ixodes TROSPA in tick colonization suggests this protein as a promising component of a vaccine to prevent the transmission of $B$. burgdorferi. The anti-TROSPA antibodies present in animal blood would compete with Borrelia for TROSPA binding in the tick gut and consequently by impeding tick gut colonization by spirochetes restrict their prevalence. The use of TROSPA in a vaccine is strongly supported by reports showing that the colonization of ticks by spirochetes was significantly impaired when the parasites fed on Borrelia-infected mice that had been injected with TROSPA antisera. Additionally, the repression of TROSPA gene expression by RNA interference significantly reduced $B$. burgdorferi adherence to the gut of $I$. scapularis [11].

The evidence presented above revealed the importance of the TROSPA protein to the Borrelia life cycle and showed that one can limit Borrelia transmission by inhibiting TROSPA-OspA binding. Accordingly, our studies were focused on exploring the details of the interaction between these proteins. Earlier this issue was investigated using TROSPA from I. scapularis and OspA from $B$. burgdorferi s. s., both typical for North America. Considering the fact that bacterial outer surface proteins may significantly differ depend on Borrelia geographical localization, we decided to use in our experiments TROSPA from $I$. ricinus, the prevalent vector of B. burgdorferi s. $l$. in Europe, and OspA from three bacterial species also typical for Europe: B. garinii, B. afzelii and B. burgdorferi s. s. We cloned TROSPA gene from I. ricinus and three OspA genes from above mentioned Borrelia species and elaborated on the methods of the production of these proteins in a bacterial system. We showed that the recombinant TROSPA was able to form complexes with its bacterial partners, three OspA proteins. Interestingly, we observed that OspA proteins from different bacterial species showed various capacities to bind TROSPA. To find out which part of TROSPA is involved in interaction with OspA and what is the nature of this interaction, we generated a series of TROSPA mutants and assessed their ability to bind OspA. Finally, we determined the immunogenic properties of recombinant TROSPA by using it to induce an immune response in rats.

\section{Results}

\section{TROSPA Expression in Bacterial and Plant Systems}

In the first stage of our studies, we attempted to produce TROSPA protein in bacterial and plant cells. To this end, the fragment of the TROSPA gene lacking the $3^{\prime}$ and $5^{\prime}$ noncoding sequences was amplified via PCR using I. ricinus genomic DNA and primers that were designed based on the reference sequence (EU384705). The PCR product was $977 \mathrm{nt}$ long, as expected. We have already submitted this sequence to GenBank (accession number KF041821). It encompassed two exons and a centrally located intron and showed $98.97 \%$ identity (ClustalW) with the reference sequence. The reference and the amplified fragment of the TROSPA gene differed by 10 nucleotides (Fig. 1A). Eight of these were located in the intron and two were within the $5^{\prime}$ exon, but the resulting amino acid sequence remained unchanged.
The predicted amino acid sequence of TROSPA contained a few potential posttranslational modification signals, including Oand C-glycosylation [11,12]. To obtain posttranslationally modified and unmodified forms of the protein, constructs for the production of TROSPA in plants and in bacteria were prepared. First, the amplified fragment of the TROSPA gene was cloned into a binary vector (pGreenII) under the control of a $35 \mathrm{~S}$ promoter. The obtained plasmid pGT was used for the transient expression of TROSPA in $\mathcal{N}$. benthamiana. Five days after agroinfiltration, total RNA was extracted from the plants, and the TROSPA cDNA was amplified by RT-PCR. Sequencing of the cDNA confirmed the correct splicing of the TROSPA sequence in $\mathcal{N}$. benthamiana (Fig. 1B). The cDNA was then cloned into a pET200 expression vector, which was used for TROSPA production in a bacterial system (Fig. 1C). The expected molecular mass of the recombinant TROSPA protein (calculated based on its amino acid composition) is about $16 \mathrm{kDa}$. A similar molecular mass has been earlier calculated for recombinant TROSPA from I. scapularis [11]. The MALDI-TOF MS analysis (data not shown) demonstrated that the molecular mass of recombinant TROSPA from $I$. ricinus is $16,1 \mathrm{kDa}$. Thus the obtained result was consistent with the above calculation. Interestingly, we found that recombinant TROSPA migrated together with $20-25 \mathrm{kDa}$ proteins when separated by SDS-PAGE (Fig. 1C). Similar phenomenon was also reported for recombinant TROSPA from I. scapularis [11].

Recombinant TROSPA, after purification on a Ni-NTA column and removal of the N-terminal tag, was used to generate TROSPA antisera. The sera were used for the detection of TROSPA in the total protein extract from agroinfiltrated fragments of $\mathcal{N}$. benthamiana leaves. Protein was isolated from plants at 6, 8, and 10 days after agroinfiltration [16]and subjected to western blot analysis. Surprisingly, TROSPA protein was not detected in $\mathcal{N}$. benthamiana leaves (data not shown).

\section{Interaction of TROSPA from I. ricinus with OspA from Borrelia burgdorferi}

As mentioned above, the I. scapularis TROSPA protein is required for tick colonization. During this process, TROSPA, located in the tick gut, specifically binds the OspA protein anchored in the B. burgdorferi outer membrane [11]. Accordingly, we predict that the TROSPA homolog from I. ricinus is also capable of binding to OspA. To verify this hypothesis, we assessed the interactions between recombinant TROSPA from $I$. ricinus and OspA from B. burgdorferi sensu stricto, B. garinii or B. afzelii. Serial dilutions of OspA preparations were added to TROSPA-coated ELISA microplates. The most significant differences between the bindings of the three OspAs to TROSPA were observed at OspA concentrations of $30 \mu \mathrm{g} / \mathrm{ml}$ (data not shown). The level of OspA binding was determined using rabbit Borrelia-specific polyclonal IgG (primary antibody) and AP-conjugated anti-rabbit polyclonal IgG (secondary antibody). Western blot analysis previously demonstrated that the primary antibody has equal capacity to bind with all three types of OspA (data not shown). Absorbance of the soluble product of the AP-catalyzed reaction was measured with reference to the blank samples that contained no OspA. The results of these assays showed a concentration-dependent formation of the OspA-TROSPA complex. Moreover, we observed that the OspA proteins derived from various Borrelia species have different abilities to bind I. ricinus TROSPA (p $\leq 0,0003$ ) (Fig. 2). As a control, analogous assays with $\mathrm{OspC}$ from $B$. garinii were 
A

KF041821 ATGGCGGCTA TGGAGGCTAT GGCGGTGGAT ATGGAGGCTA TGGCGGCGGC TATGGCGGCG GCTATGGTGG EU384705 ATGGCGGCTA TGGAGGCTAT GGCGGTGGAT ATGGAGGCTA TGGCGGCGGC TATGGCGGCG GCTATGGTGG AY640046* ATGGTGGCTA TGGAGGCTAT GGCGGC---T ATGGAGGTTA TGGTGGCGGC TATGGCGG-- -CTACGG---

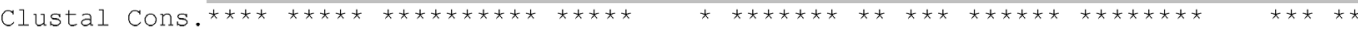
75
85
$95-105$
115
125
135

KF041821 CTACGGACAC GGTGGCTTCC TCGGCGGCTT CGGCTATGGC CACGGAGGCT ACGGTGGCTA TGGACACGGC EU384705 CTACGGACAC GGTGGCTTCC TCGGCGGCTT CGGCTATGGC CACGGAGGCT ACGGTGGCTA TGGACACGGC Clustal cons --CGGACAC GGTGGCTTCC TCGGCGGCTT CGGCTACGGC CACGGTGGCT ACGGAGGCTA TGGACACGGC
145
155
165
175
185
195
205

KF041821 GTCGCTGTCG CTGCCGCTCC AGTTGTCGCC AAGGTCGCTG CCCCAGTCGT CGCTGTCGGC CACGGCGGCC

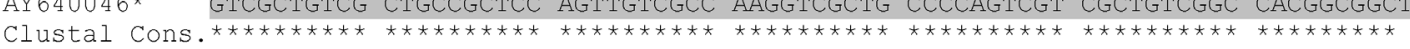

KF041821 ACGGTGGCTA CGGACACGGT GGTTTCCTCG GCGGATACGG AGGTTACGGA CACGGAGGAT TCGGCGGCTA

EU384705 ACGGTGGCTA CGGACACGGT GGTTTCCTCG GCGGATACGg AGGTTACGGA CACGGAGGAT TCGGCGGCTA

AY $640046^{\star}$ ACGGTGGCTA CGGACACGGT GGTTTCCTCG GCGGATACGG AGGTTTCGGA CACGGAGGAT TCGGCGGCTA

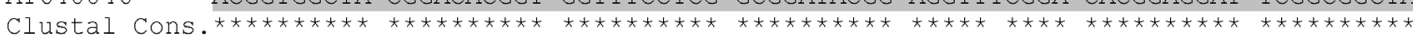
285
295
305
315
325
335
345

KF041821 CGGTCTCGGC CACGGCGTCG CTGTCCATGC TGCCCCAGTT GTCGCCAAGG TCGCTGCCCC AgTCGTCGCT

EU384705 CGGTCTCGGC CACGGCGTCG CTGTCCATGC TGCCCCAGTT GTCGCCAAGG TCGCTGCCCC AGTCGTCGCT AY640046* CGGTCTCGGG CACGGCGTTG CTGTCCATGC TGCCCCAGTC GTCGCCAAGG TCGCTCCCCC AGTCGTCGCT

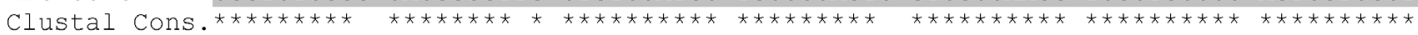
$\begin{array}{llllll}355 & 365 & 375 & 385 & 395 & 405\end{array}$

GTCGGCCACG GC---TACGg AgGCTTCGGT TACAGCGGAT ATGGCGGACA CGGCTACGTA AGTCAAATTC EU384705 GTCGGCCACG GC---TACGG AGGCTTCGGT TACGGCGGAT ATGGCGGACA CGGCTACGTA AGTCAAATTC AY640046* GTCGGCCACG GCGGCTACGG TGGATTCGGT TAC---GGAT ATGGCGGACA CGGCTATGTA AgTTAAATTC

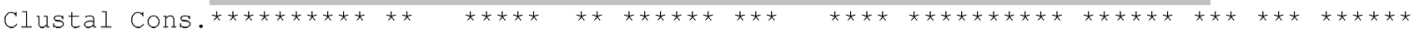
425
435
445
455
475
485

KF041821 AAAGCTATAT GGTGGTTGGC AATGTTGAAA TCTGTCTCCT TT ETGGCCCT GGAATCTGGA TTCACAGTGT EU384705 AAAGCTATAT GGTGGTTGGC AATgTTGAAA TCTGTCTCCT TTATGGCCCT GGAATCTGGA TTCACAGTGT AY640046* TAAGCTATAT GGTGGTTGGC ATGGTTGAAA TCTGTCTCCT TTAAGCCTCT GGAATCTAGA TACACAGTGT

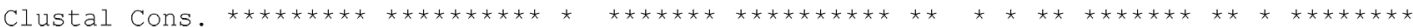
495
505
515
525
535
545
555

KF041821 GTCTTTAAGA GACGACGTAA ACATCGGCCT AATGGGCTTC TGGTGACAAC ACTTGTCCCT ATTGTCACCA EU384705 GTCTTTAAGA GACGACGTAA ACATCGGCCT AATGGGCTTC TGGTGACAAC ACTTGTCCCT ATTGTCACCA AY640046* GTCTCTGAgA GACGACGTAA ACATCGTTCT AATGGGCTTC TGGTGACAAA ACTTGTCCCT ATTGTCACCA

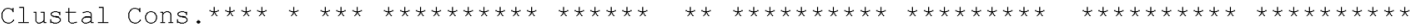

KF041821 CAACGGCCAT CATAATCTCT GATGATGCCA TTCTCATGTA GCTTATAGTC ACAGGTCGTC ATATGTGAGC EU384705 CAACGGCCAT CATAATCTCT GATGATGCCA TTCTCGTGTA GCTTATAGTC ACAGGTCGTC ATATGTGAGC AY640046* CAACGGCCAT CATAATCTCT GATGATGCCA TTATCGTATA GCTTATAATC ACAGGTTGTC ATATgtgagA

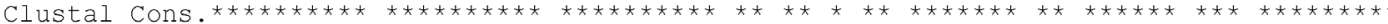
635
645
655
665
675
685695

KF041821 ACGTTGTTCA CAGCGAATGC CCATATGGA CTTCCACTTG TCGTCTGCTA AgAGCAACGC TCTAAGATGg EU384705 ACGTTGTTCA CAGCGAATGC CCATATGGAG CTTCCACTTG TMGTCTGCTA AGAGAAACGC TCTAAGATGG AY640046* ACGTTATTCA CAgCGAATGC TCATATGGAg CTTCCACTTG TCGTCTGCTA AgAGCAACGC TCTAAGATGG

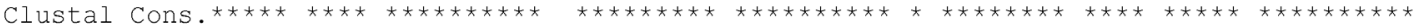
705
715
725
735
755
765

KF041821 CGTGGTATGC ACGCTTGAgA AACCTTGTGG AATCTCGCCG CCAGA TACG CTCAACCGCA ACGTCCTCAG EU384705 CGTGGTATGC ACGCTTGAGA AACCTTGTGG AATCTCGCCG ICAGAATACG CTCAACCGCA ACGTCCTCAG AY640046* CGTGGTATGC ACGCTTGGAA AACCTTGTGG AATCTTGCCG TCAGCATACG CTCAACAgCA ACGTCCTCAG

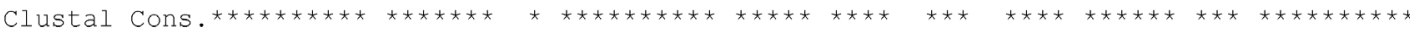
785
795
805
815
835

775

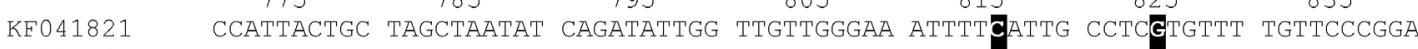
EU384705 CCATTACTGC TAgCTAATAT CAGATATTGG TTGTTGGGAA ATTTTGATTG CCTCATGTTT TGTTCCCGGA

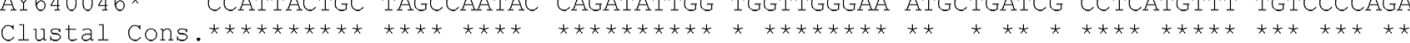
845
855
$865 \quad 875$
885
895
905

KF041821 GTGTTGGTGT TTTAACATTA TTTTTTCCTT TCTGTCTTCT TTGCAGGGAC ACTAAGCAAT TCATCTCAAA EU384705 GTGTTGGTGT TTTAACATTA TTTTTTCCTT TCTGTCTTCT TTGCAGGGAC ACTAAGCAAT TCATCTCAAA

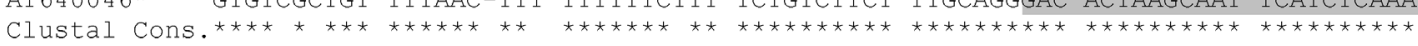
915
925
935
945
955
965
975

KF041821 GGGAAACAA CACTTCTTCG CCGCTTCTTA TTTATGCGCT TGGGCCGACC AGAGCGCCGC TGGAAGTTGA EU384705 GGGAAACCAA CACTTCTTCG CCGCTTCTTA TTTATGCGCT TGGGCCGACC AGAGCGCCGC TGGAAGTTGA AY640046* GGGAAACGAA CACTTCTTCG CCGCTTCTTA TTTATGCGCT TGGGCCGACC AGAGCGCCGC TGGAAGTTGA

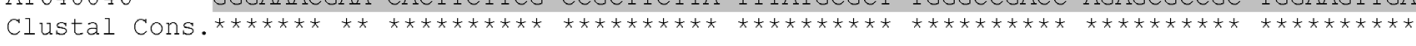
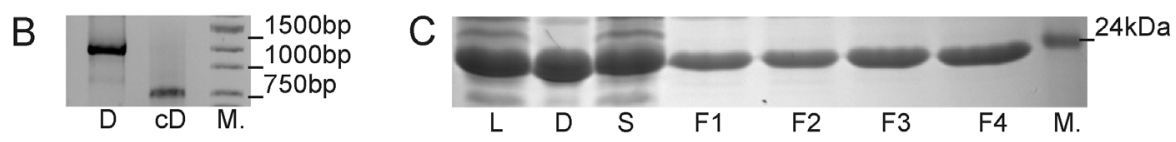
Figure 1. I. ricinus TROSPA coding sequence and the product of its expression in E. coli. A - Comparison of the DNA sequence encoding I. ricinus TROSPA KF041821 (Polish strain), reference sequence GB number EU384705 and a part of I. scapularis TROSPA gene GB number AY640046 (AY640046* - coding regions with intron are presented). The coding regions are marked in grey, the identical nucleotides are indicated with asterisks, different nucleotides are indicated with black boxes and white letters (with the respect to I. ricinus sequences). B - Image of PCR-amplified I. ricinus TROSPA DNA (D) and CDNA (CD) resolved in an agarose gel. M - DNA weight marker. C - Image of recombinant I. ricinus TROSPA protein preparations resolved by SDS-PAGE. L - E. coli lysate, D - cell debris after centrifugation of the lysate, S - supernatant after centrifugation of the lysate, F1-F4 fractions washed out from the Ni-NTA column, $\mathrm{M}$ - weight marker. doi:10.1371/journal.pone.0076848.g001

performed. These assays demonstrated no specific OspCTROSPA binding.

\section{Search for TROSPA Motifs Critical for TROSPA-OspA Interaction}

A bioinformatics analysis of the TROSPA amino acid sequence indicated that an $\mathrm{N}$-terminal transmembrane domain is present in proteins from I. scapularis and I. persulcatus $[11,12]$. Thus, it is not likely that this domain is involved in the interaction with OspA. In addition, there are indications that TROSPA-OspA interactions are electrostatic in nature; the overall charge of TROSPA protein is -12 and for OspA is +5 (in PBS, pH 7,4). To verify if these observations also apply to I. ricinus TROSPA and to identify which amino acid residues are important for interaction with OspA, two series of mutants were created. The first series included two Nterminal deletion mutants: TROSPA_N $\Delta 24$ mutant lacking amino acid 1-24 and TROSPA_N $\Delta 44$ lacking amino acids 144. A third mutant was devoid of amino acids from both the Nand C-termini: TROSPA_N $\Delta 50 \_$C $\Delta 7$ lacking amino acids 1-50 and 159-165 (Fig. 3A). The $\overline{7}$ amino acid long C-terminal fragment of TROSPA_N $\Delta 50 \_\mathrm{C} \Delta 7$ was removed due to its tendency to spontaneously degrade during the purification procedure. The degradation process was confirmed by MALDITOF MS analysis. The second series (TROSPA V1-V5) included mutants with substitutions distributed in different regions of the TROSPA protein. In these mutants, amino acid residues bearing

comparison of the level of OspA

from different Borrelia species bound to TROSPA

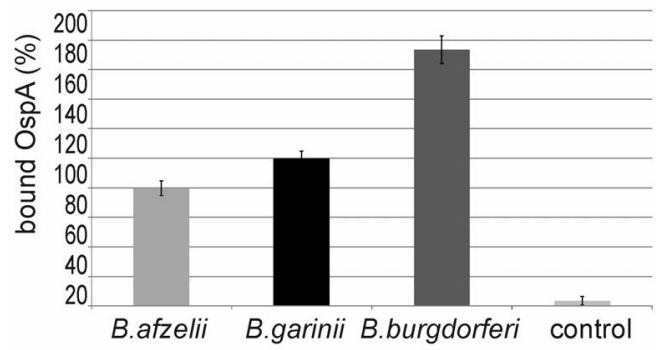

Figure 2. Comparison of the relative level of OspA protein from different Borrelia species bound to TROSPA. TROSPA proteincoated ELISA microplates were incubated with OspA protein ( $c=30 \mu \mathrm{g} /$ $\mathrm{ml}$ ) from B. afzelii, B. garinii or B. burgdorferi s. s. Bound OspA was subsequently detected using rabbit Borrelia-specific polyclonal IgG (primary antibody) and AP-conjugated anti-rabbit polyclonal IgG (secondary antibody). Absorbance of the soluble product of the alkaline phosphatase reaction was measured with reference to blank samples containing no OspA. As a control, analogous assays with OspC from $B$. garinii were performed. The level of individual OspA protein binding was always determined based on 16 reactions (for details see Materials and methods). For each protein pair arithmetic mean of absorbance and $S D$ was calculated and expressed in percentage, assuming that the level of TROSPA binding with OspA from B. garinii is $100 \%$. The differences between bound OspA from $B$. garinii and OspA from $B$. afzelii $(p=0,0003)$ or OspA from B. garinii and OspA from B. burgdorferi s. s. $(p<0,0001)$ were statistically significant. doi:10.1371/journal.pone.0076848.g002 negative charge were replaced with their neutral analogs. In the TROSPA V1 mutant, D87 and D90 were replaced with $\mathrm{N}$, and E89 was replaced with Q; in the TROSPA V2 mutant, E123 was replaced with $\mathrm{Q}$ and $\mathrm{D} 129$ was replaced with $\mathrm{N}$; in the TROSPA V3 mutant, D132 and D136 were replaced with $\mathrm{N}$; in the TROSPA V4 mutant, D75, D82, D87 and D90 were replaced with $\mathrm{N}$, and E84 and E89 were replaced with Q; in the TROSPA V5 mutant, E123 was replaced with Q and D129, D132, D136 and D159 were replaced with N (Fig. 3B).

All of these mutants were expressed in a bacterial system and purified as previously described for wild type protein. The influence of the mutations on the ability of TROSPA to bind OspA was determined by immunoassay. Microplates were coated with TROSPA mutants $(\mathrm{C}=5 \mu \mathrm{g} / \mathrm{ml}, 100 \mu \mathrm{l} /$ well $)$ and probed with $B$. garinii OspA at a concentration of approximately $30 \mu \mathrm{g} / \mathrm{ml}$ (100 $\mu \mathrm{l} /$ well). B. garinii OspA was chosen because among the three previously tested OspA proteins, it showed a moderate level of affinity for TROSPA. The results (Fig. 3) revealed that deletion of the N-terminal 24 or 44 amino acids of TROSPA, or deletion of the whole predicted transmembrane domain $(50 \mathrm{~N}$-terminal amino acids) together with 7 amino acids from the C-terminus, slightly increased the capacity of TROSPA to bind OspA (by $11 \%$, $17 \%$ and $13 \%$, respectively, compared to wt TROSPA; $\mathrm{p} \leq 0,0003$ ) (Fig. 3G). In contrast, the mutants bearing the reduced total negative charge displayed significantly lower affinity for OspA (by $31 \%, 25 \%, 24 \%, 23 \%$ and $39 \%$ for V1-V5 mutants, respectively; $\mathrm{p}<0,0001)$ compared to control wt TROSPA. However, none of the V1-V5 series of mutants showed a complete loss of binding affinity for OspA.

\section{I. ricinus TROSPA as a Candidate for a Vaccine Against Borreliosis}

TROSPA protein has been considered a potential antigen for a vaccine against borreliosis $[11,12,17]$. It was shown that in animals experimentally infected with Borrelia, administration of $I$. scapularis TROSPA antisera reduced (up to $75 \%$ ) the colonization of the tick and subsequently the number of spirochetes that were transmitted to the next mammalian host [11].

In our studies, TROSPA antiserum, generated using recombinant $I$. ricinus TROSPA, was used to examine the influence of antiTROSPA immunoglobulin on the formation of the TROSPAOspA complex. The experiments were conducted similarly for the TROSPA mutants. However, before OspA was added to the TROSPA-coated microplates, the plates were treated with serial dilutions of I. ricinus TROSPA antiserum (Fig. 4). In a control experiment, TROSPA-coated microplates were treated with preimmune rabbit serum. After plates were incubated with $B$. garinii OspA protein, the level of OspA binding with TROSPA was determined using FITC-conjugated anti-Borrelia IgG and fluorescence counting. TROSPA-coated plates treated only with FITCconjugated anti-Borrelia IgG were used as reference samples. As expected, significantly less OspA was bound to the TROSPAcoated microplates treated with TROSPA antiserum compared to microplates treated with control preimmune serum. A $30 \%$ reduction of TROSPA-OspA binding was observed when 
A

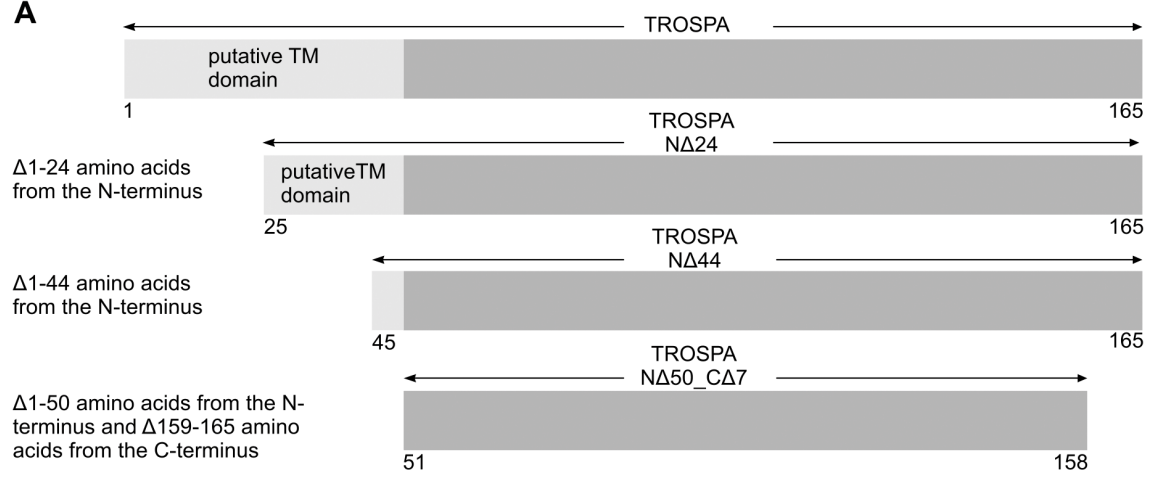

B

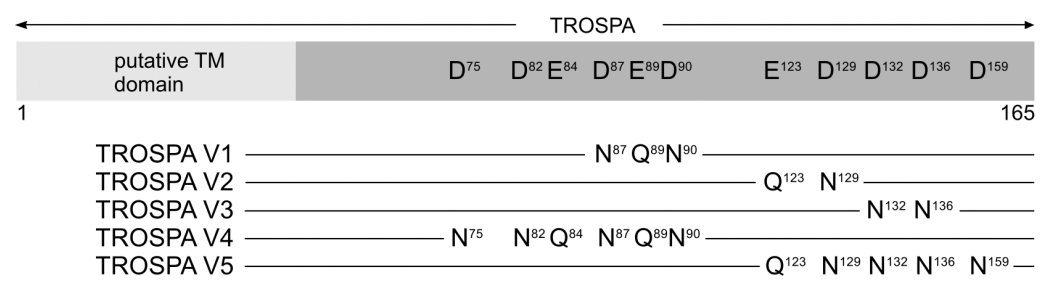

C

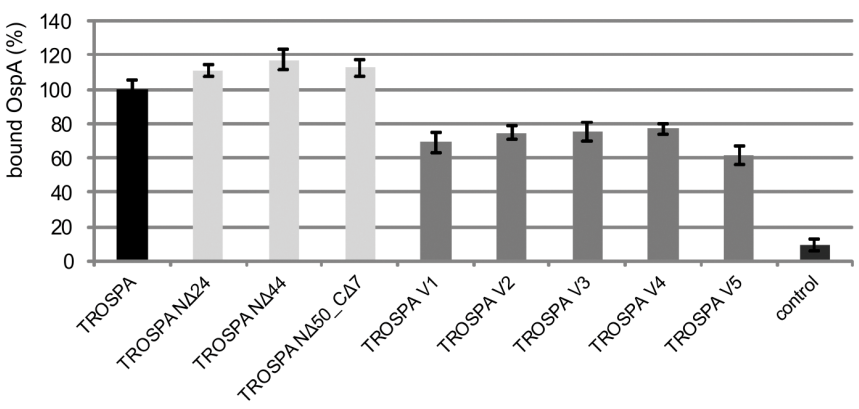

Figure 3. Schematic depiction of the two series of I. ricinus TROSPA mutants and the level of bound OspA protein from Borrelia garinii. A - TROSPA deletion mutants. In the N $\Delta 24$ mutant, amino acids 1 to 24 were deleted; in the N $\Delta 44$ mutant, amino acids 1 to 44 were deleted; in the N $\Delta 50 \_C \Delta 7$ mutant, amino acids 1 to 50 and 159 to 165 were deleted. B - TROSPA substitution mutants. Selected negatively charged amino acid residues were changed to neutrally charged amino acids: in the V1 TROSPA mutant, aspartic acids 87 and 90 were replaced with asparagine, and glutamic acid 89 was replaced with glutamine; in the V2 TROSPA mutant, glutamic acid 123 was replaced with glutamine, and aspartic acid 129 was replaced with asparagine; in the V3 TROSPA mutant, aspartic acids 132 and 136 were replaced with asparagines; in the V4 TROSPA mutant, aspartic acids 75, 82, 87 and 90 were replaced with asparagines, and glutamic acids 84 and 89 were replaced with glutamines; in the V5 TROSPA mutant, glutamic acid 123 was replaced with glutamine, and aspartic acids 129, 132, 136 and 159 were replaced with asparagines. C - Comparison of the relative level of OspA from B. garinii bound to the TROSPA mutants. TROSPA- or TROSPA mutants- coated ELISA microplates were incubated with $B$. garinii OspA protein $(C=30 \mu \mathrm{g} / \mathrm{ml}, 100 \mu \mathrm{l} /$ well). Bound OspA was subsequently detected using rabbit Borrelia-specific polyclonal IgG (primary antibody) and AP-conjugated anti-rabbit polyclonal lgG (secondary antibody). Absorbance of the soluble product of the alkaline phosphatase reaction was measured with reference to blank samples containing no OspA. For each TROSPA or TROSPA mutant the level of OspA binding was determined based on 16 reactions (for details see Materials and methods). For each protein pair arithmetic mean of absorbance and SD was calculated and expressed in percentage, assuming that the level of TROSPA binding with OspA from B. garinii is $100 \%$. The differences of OspA binding among unmutated TROSPA and the deletion mutants were statistically significant ( $p=0,0002$ for the N $\Delta 24$ mutant, $p<0,0001$ for the N $\Delta 44$ mutant and $p=0,0003$ for the $\mathrm{N} \Delta 50 \_\mathrm{C} \Delta 7$ mutant). All the mutants bearing the reduced total negative charge (the V1-V5 mutants) displayed significantly lower affinity for OspA $(p<0,0001)$ compared to control unmutated TROSPA. doi:10.1371/journal.pone.0076848.g003

TROSPA antiserum was applied at the highest concentration $(\mathrm{p}=0,001)$.

To further examine the antigenic properties of recombinant TROSPA protein from I. ricinus, we used TROSPA for oral immunization of animals. Two types of immunizations were performed, with the use of the TROSPA protein alone or with the use of the TROSPA protein together with B. garinii OspA and OspC proteins. A combined vaccine composed of these three proteins would generate the antibodies working against Borrelia infection on different stages of its life cycle. We wanted to find out if the application of three different antigens together would influence the level of the generated anti-TROSPA antibodies. The first group of 10 rats was immunized with TROSPA protein (including 5 individuals immunized with the addition of an adjuvant). The second group of 10 rats was immunized with TROSPA protein in combination with B. garinii OspA and OspC proteins, including 5 individuals immunized with the addition of an adjuvant. The experiments with or without adjuvant were performed to address the question to what extent the immunogenic properties of TROSPA would be enhanced by adjuvant addition. The control group of 5 rats was immunized with the adjuvant alone. Rats were immunized three times, on days 0,14 , 


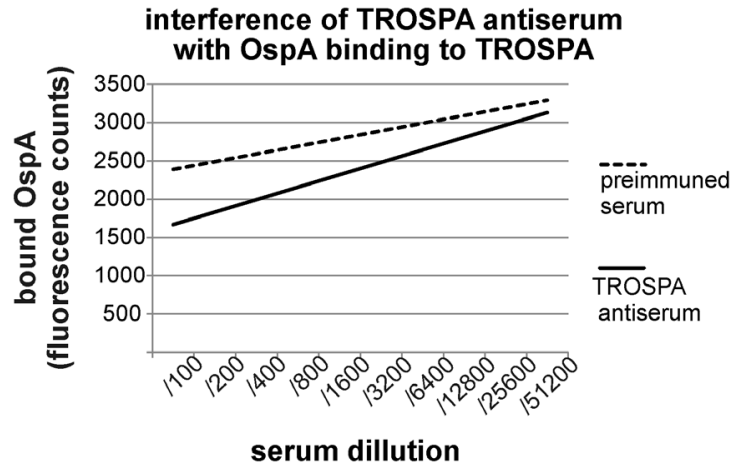

Figure 4. Interference of the TROSPA antiserum with the level of OspA bound to TROSPA. I. ricinus TROSPA protein-coated ELISA microplates were incubated with the serial dilutions of rabbit TROSPA antiserum or rabbit preimmune antiserum. After washing, the plates were treated with $B$. garinii OspA protein $(C=30 \mu \mathrm{g} / \mathrm{ml}, 100 \mu \mathrm{l} /$ well), which was subsequently detected by FITC-conjugated anti-Borrelia goat polyclonal IgG. The $X$ axis represents serial sera dilutions, and the $Y$ axis represents the level of fluorescence counts. The level of TROSPA-OspA binding was determined for ten rabbit TROSPA antiserum or preimmune serum concentrations. The level of OspA binding was determined as the arithmetic mean of fluorescence counts based on 16 reactions performed for every TROSPA antiserum or preimmune serum dilution (for details see Materials and methods). The reduction of fluorescence counts was statistically significant $(p<0,01)$ for all serum dilutions except the highest one.

doi:10.1371/journal.pone.0076848.g004

and 28. During the experiment, the rats were observed and weighed every seven days to exclude negative effects of immunization on their health. After 42 days, serum sampling was performed. Sera were serially diluted and an ELISA was used to assess the level of induced IgG (Fig. 5). In both groups of animals, high levels of anti-TROSPA IgG were detected, although the levels of anti-OspA and anti-OspC IgG were significantly higher $(p=0,001)$. The level of anti-TROSPA IgG was defined as the biggest reciprocal serum dilution which showed higher absorbance readings than the control serum in the ELISA [18]. In TROSPAimmunized or TROSPA-, OspA- and OspC-immunized animals, the level of anti-TROSPA IgG reached 2500 (without adjuvant) or 5000 (with adjuvant). The levels of anti-OspA and anti-OspC IgG (also defined as the biggest reciprocal serum dilution showing higher absorbance than the control) were as high as 10000 .

\section{Discussion}

To initiate circulation in the natural environment, B. burgdorferi colonizes ticks [11]. One of the key factors involved in this process is a tick gut protein, TROSPA. TROSPA retains bacteria in the vector digestive tract by binding the spirochete surface protein $I$. ricinus is the widest spread vector of Borrelia in Europe [1,4,7,8,19]. Accordingly, the main object of our studies was the TROSPA protein from this species. First, we amplified and cloned a fragment of the TROSPA gene. This fragment contained two exons encoding whole TROSPA and one intron; the cloned fragment was nearly identical $(98.97 \%)$ with the reference sequence EU384705. The amino acid sequences deduced from both amplified and reference DNA sequences were identical (Fig. 1A). Next, we attempted to produce TROSPA protein in plant and bacterial expression systems. We found that TROSPA was efficiently produced in the $E$. coli cells, while it was not detected in the total protein isolated from the plant cells. However, the presence of correctly spliced mRNA was confirmed (Fig. 1B).
TROSPA was detected on western blots using sera generated in animals injected with recombinant protein that was produced in bacteria. Earlier studies of TROSPA isolated from I. scapularis suggest that this protein is extensively glycosylated $[11,12]$. Accordingly, it could be that TROSPA was undetected in plants because the antibodies were targeted against non-glycosylated protein. This is most likely not the case because the above mentioned study showed that antisera raised against recombinant TROSPA (from I. scapuaris) produced in E. coli specifically reacted with the glycosylated native TROSPA protein from I. scapularis gut cell lysates [11]. Thus, we reason that native TROSPA from $I$. ricinus should also be detected with antisera raised against recombinant protein. Accordingly, we propose that TROSPA was not effectively produced in our plant system or that it was produced and then rapidly degraded by plant proteases. Similar phenomena have been reported for many other bacterial or animal proteins that were expressed in various plant systems [20].

We showed that highly purified, non-glycosylated recombinant I. ricinus TROSPA can be obtained using affinity chromatography. Next, we examined if this protein retains its natural capacity to bind bacterial OspA. The most abundant Borrelia species in Europe are: B. burgdorferi sensu stricto, B. garinii and B. afzelii [1], thus, in our experiments we used recombinant OspA proteins derived from these species. We observed that the TROSPA-OspA complex was formed in all cases; however, the amount of complex was slightly different for the three proteins (Fig. 2). We conclude that $I$. ricinus TROSPA can be involved in tick colonization by at least these three species of Borrelia, however the efficacy of colonization may varies depending on Borrelia species. Similar observations have been made by other researchers. For example, $\mathrm{Pal}$ and coworkers reported that OspA from B. burgdorferi s. s. strains N40 and 25015 were both able to bind to the tick gut extract, but the 25015 OspA formed a more stable complex [21]. If there is such a variation within one bacterial species, the differences observed for OspA derived from different species are not surprising. The question is whether these differences result only from OspA-TROSPA binding or also from interactions between OspA-OspA. It was demonstrated that OspA proteins are capable of forming homopolymeric complexes [21]. In this way, the number of the OspA proteins, and consequently bacterial cells, that are attached to the tick gut epithelial cells can be increased. Taking into account the conservation of TROSPA and the variability of OspA, one can hypothesize that the efficiency of vector colonization by Borrelia to a higher extent depends on the properties of bacterial than tick encoded protein. In other words, it is possible that the type of OspA may influence the local prevalence of Borrelia species.

So far, the molecular determinants of TROSPA-OspA recognition remain unknown. To investigate the nature of the TROSPA-OspA interaction, we prepared several TROSPA mutants. Bioinformatics analysis of the TROSPA protein amino acid sequence indicated the presence of an N-terminal transmembrane domain and an overall negative charge of the protein $(-12)$. Similar analysis of OspA revealed that it carries a positive charge $(+5)$. Therefore, we hypothesized that electrostatic interactions may play a significant role in the TROSPA-OspA binding. To verify this hypothesis, we designed two series of TROSPA mutants. The first series included two mutants with an N-terminal deletion encompassing a large fragment or the whole putative transmembrane domain (Fig. 3A). The second series included mutants in which selected amino acid residues bearing negative charge were replaced with their neutral analogs (Fig. 3B). The analysis of the interaction between the TROSPA deletion mutants and OspA protein from B. garinii confirmed that the N-terminal 
A level of anty-TROSPA IgG
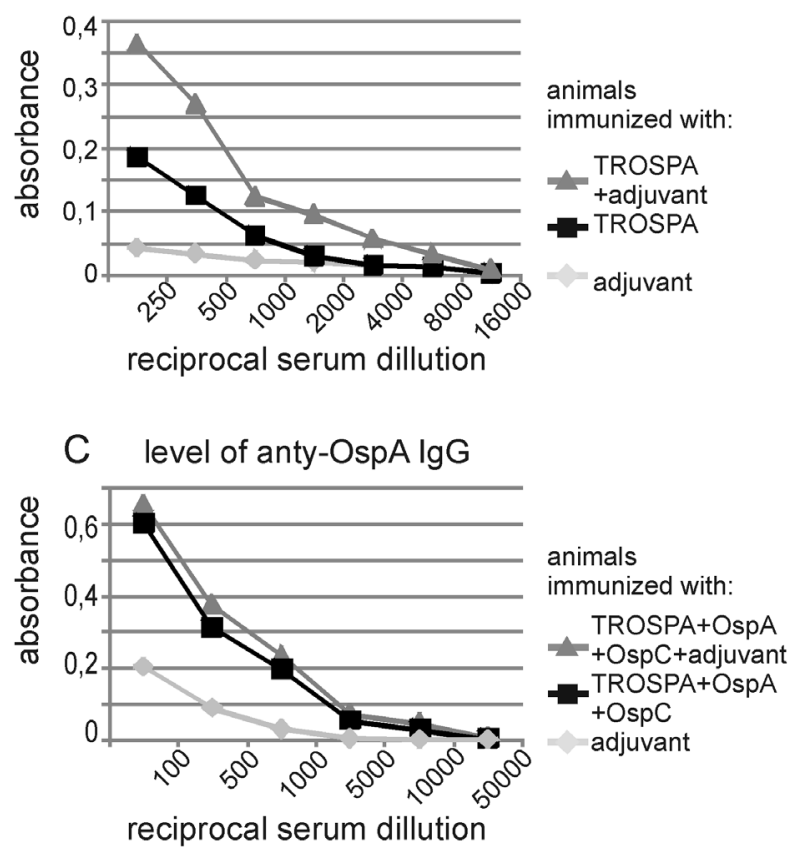

\section{B level of anty-TROSPA IgG}
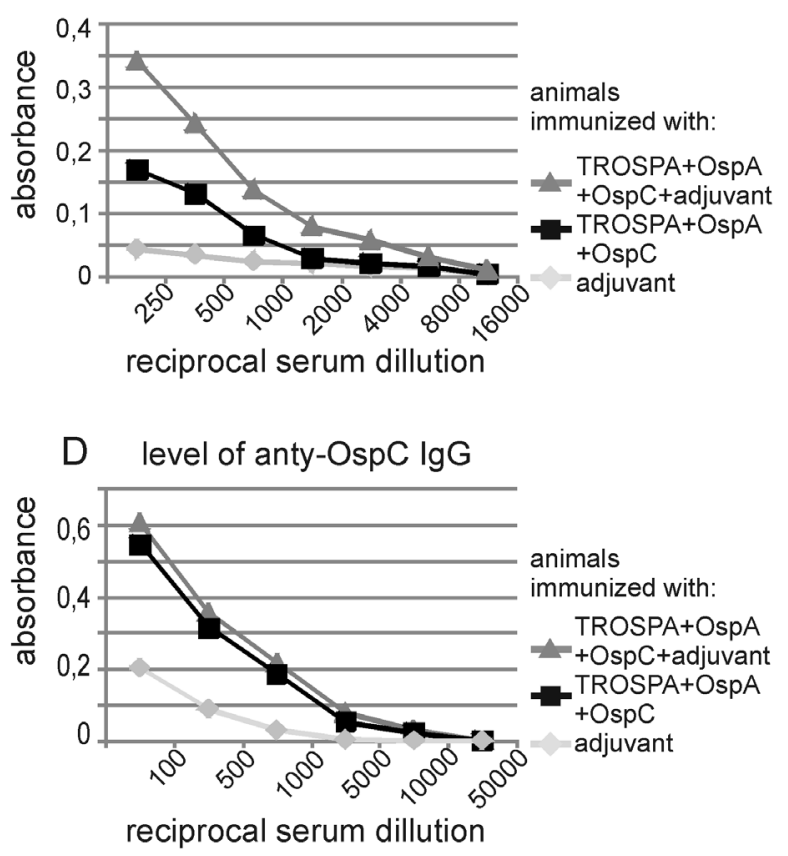

Figure 5. Titers of IgG detected by ELISA in the sera of orally immunized rats. Rats were immunized on days 0,14 , and 28 ; sera were collected on day 42. Next, sera were serially diluted and subjected to ELISA tests. A - The level of specific lgG in groups of 5 rats immunized with TROSPA or TROSPA with adjuvant. B-D - The level of specific IgG in groups of 5 rats immunized with TROSPA, OspA and OspC without or with adjuvant. The control group of 5 rats was immunized with adjuvant only. The $X$ axis represents the reciprocal sera dilutions, and the $Y$ axis represents the level of absorbance of the color product of the alkaline phosphatase reaction. The levels of IgG were calculated as the arithmetic means of absorbance measured in ELISA tests performed for the serially diluted sera from each group of 5 animals. The levels of IgG were determined based on 15 reactions performed for every serum dilution (for details see Materials and methods). doi:10.1371/journal.pone.0076848.g005

part of TROSPA does not play a role in OspA binding (Fig. 3G). The removal of this domain, in whole or part, did not significantly decrease the capacity of TROSPA to bind OspA. In contrast, analysis of the capacities of TROSPA V1-5 mutants to bind with OspA was not entirely conclusive. All the substitution mutants showed significantly lower affinity for OspA compared to the wild type TROSPA (Fig. 3C). However, none of the mutations caused TROSPA to be incapable of binding with OspA. Similarly, amino acid substitutions introduced by $\mathrm{Pal}$ and coworkers to OspA regions that strongly bound to tick gut extract did not definitively identify the character of the interaction [21]. Thus, the nature of the TROSPA-OspA interaction seems to be more complex than electrostatic interactions alone. Because the native TROSPA protein can be post-translationally modified, one cannot exclude that these modifications also participate in these interactions. To examine this possibility, the stability of the complexes formed by native and recombinant TROSPA with OspA should be compared.

In Europe, OspA shows a significant level of diversity [1,19,22], this being one of the reasons underlying the ineffective diagnosis and prevention of Lyme disease. In contrast to OspA, I. ricinus TROSPA seems to be a very conservative protein, taking into account GenBank data (Fig. S1). In addition, TROSPA clearly binds with OspA from different strains of Borrelia, a crucial step in the spirochete life cycle. These features of TROSPA make the $I$. ricinus protein a good candidate for a vaccine against Borrelia. We propose that a vaccine containing this protein can be used for the immunization of wild animals, which are the natural reservoir for the bacteria. This would serve to lower the infection rates of Ixodes ticks and consequently reduce the risk of Borrelia transmission to humans. Our studies also showed that oral immunization of animals with recombinant $I$. ricinus TROSPA protein induced a high level of IgG (Fig. 5), suggesting strong immunogenic properties of this protein [23]. We further demonstrated that antibodies against TROSPA can outcompete OspA protein in binding with TROSPA (Fig. 4).

In conclusion, the data collected here indicate that recombinant TROSPA protein from I. ricinus produced in a bacterial system retains the ability to interact with OspA from three most abundant European species of Borrelia. The different affinity of TROSPA to OspA derived from various bacterial species suggests that this interaction may influence the occurrence of particular Borrelia species in a given geographic area. The N-terminal part of TROSPA is not involved in the interaction with OspA, which is consistent with in silico predictions. However, further studies are required to precisely determine the amino acids or structural motifs that are involved in forming TROSPA - OspA complex, because it is only partially determined by the electrostatic interactions. Finally, I. ricinus TROSPA protein exhibits a number of features making it a good candidate for a vaccine for the wild life in order to decrease the natural reservoir of Borrelia. These features are: conservative amino acid sequence, ability to induce a high level of antibodies in orally immunized animals and ability to compete with OspA for TROSPA binding.

\section{Materials and Methods}

\section{Ethics Statement}

The experiments involving animals were performed in strict accordance with standards of European Union legislation. The 
protocols were evaluated and approved by the Local Ethical Committee for Animal Experimentation in Poznan, Poland (Permit Number: 52/2008). The rats were anaesthetized by intramuscular injection of ketamine and xylazine (30-35 and 40$90 \mathrm{mg} / \mathrm{kg}$, respectively), all efforts were made to minimize animal suffering and to reduce the number of animals used.

\section{Construction of Plasmids for the Production of TROSPA, OspA and OspC Proteins in a Bacterial System}

I. ricinus ticks were obtained from Dr. Beata Wodecka from the Department of Genetics, Szczecin University, Szczecin, Poland. Genomic DNA of I. ricinus was extracted from the ticks using a QIAGEN DNeasy Blood \& Tissue Kit. A fragment of the TROSPA gene located between the start and stop codons (a fragment containing two exons and a centrally positioned intron) was amplified by PCR using primers $\mathrm{T}^{\prime}$ and $\mathrm{T} 3^{\prime}$. The primers were designed based on the sequence of the I. ricinus TROSPA gene (GenBank sequence number EU034646.1). The sequence of the 977-nt long PCR product was submitted to GenBank, accession number KF041821. The PCR product was ligated into the pGreenII vector using $E c o$ RI and SmaI restriction sites. The pGreenII-based construct bearing the fragment of the TROSPA gene under the $35 \mathrm{~S}-\mathrm{CaMV}$ promoter (named pGT) was used to transform the Agrobacterium tumefaciens GV3101 strain. The resultant $A$. tumefaciens GV3101-pGT strain was applied to 6-week old Nicotiana benthamiana plants for agroinfiltration. Briefly, after overnight culture, $1 \mathrm{ml}$ of A. tumefaciens GV3101-GT was centrifuged for $3 \mathrm{~min}$ at $8 \mathrm{krpm}$ at room temperature, and the pellet was dissolved in $10 \mathrm{ml}$ of infiltration buffer $(10 \mathrm{~m} M$ MES$\mathrm{NaOH}$ pH 5.5, $10 \mathrm{~m} M \mathrm{MgSO}_{4}$ ). The undersides of $\mathcal{N}$. benthamiana leaves were infiltrated with syringe. Agroinfiltrated plants were maintained at $23^{\circ} \mathrm{C}$ in a growth chamber with a 16 -h photoperiod. Five days post agroinfiltration, total RNA was extracted from $\mathcal{N}$. benthamiana using a QIAGEN RNeasy Plant Mini Kit. TROSPA cDNA was amplified by reverse transcription and PCR using TF and T3' primers and cloned into a pET vector using a Champion ${ }^{\mathrm{TM}}$ pET200 Directional TOPO Expression Kit (Invitrogen) according to the manufacturer's instructions.

DNA encoding either $B$. burgdorferi sensu sticto (ZS7 strain), $B$. afzelii or B. garinii OspA (Materials S1B) and B. garinii OspC (GB accession number: D49498.1) were provided by Dr. Beata Wodecka (Department of Genetics, Szczecin University, Szczecin, Poland). The OspA genes were amplified via PCR using primers $\mathrm{AF}$ and $\mathrm{AR}$, and the $\mathrm{OspC}$ gene was amplified using primers $\mathrm{CF}$ and CR. The PCR products were cloned into a pET expression vector using a Champion ${ }^{\mathrm{TM}}$ pET200 Directional TOPO ${ }^{\circledR}$ Expression Kit according to the manufacturer's instructions. All the primer sequences are in Materials S1A.

\section{TROSPA Mutant Preparation}

To obtain plasmids encoding TROSPA deletion mutants (TROSPA M1_A24del designated as TROSPA_ND24, TROSPA M1_M44del designated as TROSPA_N $\Delta 44$, TROSPA M1_S50 del_D159_S165del designated as TROSPA_N $\left.\Delta 50 \_C \Delta 7\right)$, the selected fragments of TROSPA cDNA were obtained by PCR using the following primers: $\mathrm{T} 24 \mathrm{~F}$ and TR to amplify DNA encoding TROSPA shortened by 24 amino acids from the Nterminus; T44F and TR to amplify DNA encoding TROSPA shortened by 44 amino acids from the $\mathrm{N}$-terminus; T50F and T7R to amplify DNA encoding TROSPA shortened by 50 amino acids from the $\mathrm{N}$-terminus and by 7 amino acids from the C-terminus. Plasmids (pET200-derivatives) containing cDNA of TROSPA substitution mutants (TROSPA V1, V2 and V3) were produced according to the Molecular Cloning mutagenesis protocol [24] using
I. ricinus TROSPA cDNA in pET200 as a template. The following pairs of primers were used for PCR to produce particular TROSPA mutants: T1F and T1R to obtain TROSPA V1, T2F and T2R to obtain TROSPA V2, T3F and T3R to obtain TROSPA V3. TROSPA V4 and V5 cDNA were ordered from the GeneArt ${ }^{\circledR}$ company and amplified by PCR using TF and TR primers. All the primer sequences are in Materials S1A. The modified TROSPA cDNAs were cloned into the pET expression vector using a Champion ${ }^{\mathrm{TM}}$ pET200 Directional TOPO Expression Kit according to the manufacturer's instructions. As a result, the following pET200 plasmids encoding TROSPA or its mutants were obtained: pET200-TROSPA, pET200-TROSPA_N $\Delta 24$, pET200-TROSPA_N $\Delta 44$, pET200-TROSPA_N $\Delta 50 \_C \Delta 7$, pET200TROSPA_V1, pET200-TROSPA_V2, pET200-TROSPA_V3, pET200-TROSPA_V4, pET200-TROSPA_V5.

\section{Protein Production and Purification}

TROSPA and its mutants were expressed in BL21 Star $^{\text {TM }}$ (DE3) One ShotR Chemically Competent E. coli according to the manufacturer's instructions. Briefly, bacterial cells were transformed with the modified pET200 plasmids (encoding wt or mutated TROSPA) by a heat shock method. Then, each transformant was used to inoculate $250 \mathrm{ml}$ of $\mathrm{LB}$ medium containing $50 \mathrm{mg} / 1$ kanamycin. The cells were grown at $37^{\circ} \mathrm{C}$ and when the $\mathrm{OD}_{600}$ of the culture reached 0.5-0.8, IPTG was added to a final concentration of $0.5 \mathrm{~m} M$. After induction, the culture was incubated for $4 \mathrm{~h}$ at $37^{\circ} \mathrm{C}$. The cell paste was harvested and frozen on dry ice for storage at $-20^{\circ} \mathrm{C}$. Approximately $10 \mathrm{~g}$ of cell paste was resuspended in $20 \mathrm{ml}$ lysis buffer I [50 mM Na $\mathrm{HPO}_{4} \mathrm{pH}$ 8, $50 \mathrm{~m} M$ NaCl, 1x CelLyticTM (Sigma-Aldrich) MT, 250U Benzonase ${ }^{\circledR}$ Nuclease (Novagen), 1 tablet of Mini EDTA-free Protease Inhibitor Cocktail Tablets (Roche), $0.2 \mathrm{mg} / \mathrm{ml}$ lysozyme (BioShop)]. After incubation on ice for $30 \mathrm{~min}$, the lysate was sonicated three times for $30 \mathrm{~s}$ on ice. Cell debris was removed by centrifugation and the supernatant was mixed with the same amount of buffer II (50 $\mathrm{mM} \mathrm{Na} \mathrm{HPO}_{4}$ $\mathrm{pH} \mathrm{7,} 300 \mathrm{~m} M \mathrm{NaCl}, 30 \mathrm{~m} M$ imidazole). To purify TROSPA and its mutants, we used affinity chromatography. After protein binding with Ni-NTA resin, the column was washed with $30 \mathrm{mM}$ imidazole in buffer III $\left(50 \mathrm{~m} M \mathrm{Na}_{2} \mathrm{HPO}_{4} \mathrm{pH}\right.$ 6, $300 \mathrm{~m} M \mathrm{NaCl}$, $5 \%(v / v)$ glycerol). Next, the protein was eluted from the column using $200 \mathrm{~m} M$ imidazole in buffer III. The eluted protein was dialyzed using PBS buffer. To remove the N-terminal polyhistidine-tag, a Enterokinase Cleavage Capture Kit (Millipore) was used according to the manufacturer's instructions. After digestion, the sample was once again applied to a Ni-NTA charged column to remove the tag and any undigested protein. The first flowthrough was collected and dialyzed in PBS buffer. The sequence of TROSPA was confirmed by MALDI-TOF MS. Finally, rabbit TROSPA-antiserum was produced by Eurogentec (according to an 87 day standard protocol) using the highly purified TROSPA protein provided by us.

\section{Analysis of TROSPA-OspA Interaction}

ELISA microplates were coated with TROSPA or TROSPA mutants solution $(\mathrm{c}=5 \mu \mathrm{g} / \mathrm{ml})$ in PBST buffer (PBS buffer containing $0,05 \%$ Tween-80) and incubated overnight at $4^{\circ} \mathrm{C}$. The microplates were washed five times with PBST using ImmunoWash (Model 1575, Bio-Rad), and the nonspecific binding sites were blocked with $3 \%$ BSA in PBST overnight at $4^{\circ} \mathrm{C}$. After another five washes with PBST, $100 \mu \mathrm{l}$ of OspA solutions (serially diluted in PBST, eleven OspA concentrations ranging from $250 \mu \mathrm{g} / \mathrm{ml}$ to $0,2 \mu \mathrm{g} / \mathrm{ml}$ ) were applied to each well except for the first vertical row that was composed of blank 
samples. In a control assay, OspC solution was used instead of OspA. The microplates were incubated for $90 \mathrm{~min}$ at room temperature and then washed five times with PBST. Next, $100 \mu \mathrm{l}$ of anti-Borrelia IgG (Abcam) diluted 1:10000 in PBST containing $1 \%$ BSA (PBSTB) was applied to each well. The microplates were incubated for $90 \mathrm{~min}$ at room temperature and then washed five times with PBST. Next, $100 \mu \mathrm{l} /$ well of AP-conjugated anti-rabbit IgG (Abcam) diluted 1:50000 in PBSTB was applied, and the microplates were incubated for $90 \mathrm{~min}$ at room temperature. Finally, the microplates were washed five times with PBST, and the color was developed at room temperature by adding $100 \mu \mathrm{l} /$ well of AP Substrate Kit (Bio-Rad). The reactions were terminated after $30 \mathrm{~min}$ by the addition of $0.4 \mathrm{M} \mathrm{NaOH}(100 \mu \mathrm{l} /$ well). Absorbance values were read at $405 \mathrm{~nm}$ in a Microplate Reader (Model 550, Bio-Rad). For each TROSPA (wild type or mutant) OspA (either from B. garinii, B. afzelii or B. burgdorferi s. s) pair the level of protein binding was determined for eleven OspA concentrations and each individual reaction was repeated 8 times. All experiments were repeated twice. Thus, for TROSPA and each TROSPA mutant the level of OspA binding was determined based on 16 reactions performed for every OspA concentration (2 biological and 8 technical replicates). For each protein pair arithmetic mean of absorbance and SD was calculated for each OspA concentration. Next, the relative level of TROSPA (TROSPA mutant) - OspA binding was calculated. To this end, the arithmetic means of absorbance obtained for all protein pairs at the same OspA concentration were divided by the arithmetic mean of absorbance calculated for OspA from B. garinii at corresponding concentration. The relative level of protein binding was expressed in percentage, accordingly, the level of TROSPA binding with OspA from B. garinii was considered as 100\%.

\section{Influence of TROSPA-antiserum on TROSPA-OspA Interaction}

TROSPA-coated ELISA microplates (prepared as described above) were incubated for $90 \mathrm{~min}$ at room temperature with $100 \mu \mathrm{l} /$ well serially diluted (in PBST) rabbit TROSPA antiserum or rabbit preimmune serum (Eurogentec). Both TROSPA antiserum and preimmune serum were diluted 100 to 51,200 times. One dilution per one microplate column including eight wells was applied. The microplates were washed five times with PBST using Biorad Immunowash at room temperature. Then, $100 \mu \mathrm{l} /$ well of B. garinii OspA solution (c $=30 \mu \mathrm{g} / \mathrm{ml}$ ) in PBST was applied and the microplates were incubated for $90 \mathrm{~min}$ at room temperature. The microplates were washed five times with PBST, and then $100 \mu \mathrm{l} /$ well of FITC-conjugated anti-Borrelia IgG (Abcam) diluted 1:500 in PBST was applied. The microplates were incubated for $90 \mathrm{~min}$ at room temperature and washed five times with PBST. Fluorescence was measured using a PerkinElmer VICTOR X4 2030 Multilabel Reader. The level of TROSPAOspA binding was determined for ten TROSPA antiserum/ preimmune serum dilutions and each individual reaction was repeated 8 times. All experiments were repeated twice. Thus, to determine the level of OspA binding the arithmetic mean of fluorescence intensity was calculated based on 16 reactions performed for every TROSPA antiserum/preimmune serum dilutions (2 biological and 8 technical replicates).

\section{References}

1. Franke J, Hildebrandt A, Dorn W (2013) Exploring gaps in our knowledge on Lyme borreliosis spirochaetes-updates on complex heterogeneity, ecology, and pathogenicity. Ticks Tick Borne Dis 4: 11-25.

\section{Immunization}

Wistar rats provided by Department of Toxicology from the Poznan University of Medical Sciences were used for the oral immunization experiments. Groups of five rats were orally immunized three times (on days 0, 14, and 28). Briefly, $200 \mu \mathrm{g}$ of the purified proteins (either TROSPA or TROSPA, OspA and OspC together) in PBS buffer with or without 1 unit of GEM particles (Gram-positive enhancer matrix, an adjuvant derived from Lactococcus lactis, [25]) were added to sodium bicarbonate solution $(3.2 \% \mathrm{w} / \mathrm{v})$ and administered to each rat. Control rats were administered PBS buffer with 1 unit of GEM particles only. The oral administration was performed using a stainless steel feeding needle. After 42 days, blood sampling was performed via cardiac puncture and sera were obtained by centrifugation and stored at $-80^{\circ} \mathrm{C}$ until further analysis.

\section{IgG Levels in Orally Immunized Rat Sera}

The serial dilutions of rat serum in PBST were applied to TROSPA-, OspA- (B. garinii) or OspC- (B. garini $)$ coated ELISA microplates (prepared as described above) and incubated for $3 \mathrm{~h}$ at room temperature. The microplates were washed five times with PBST followed by application of $100 \mu \mathrm{l} /$ well of AP-conjugated anti-rat IgG (Invitrogen) diluted 1:5000 in PBST. The microplates were incubated for $90 \mathrm{~min}$ at room temperature. Finally, plates were washed five times with PBST, and the color was developed at room temperature by adding $100 \mu \mathrm{l} /$ well of BIORAD AP Substrate Kit. The reaction was terminated after $30 \mathrm{~min}$ by 0.4 $\mathrm{M} \mathrm{NaOH}$ addition $(100 \mu \mathrm{l} /$ well). Absorbance was read at $405 \mathrm{~nm}$ in a Microplate Reader (Model 550, Bio-Rad). In order to determine the level of $\mathrm{IgG}$ the arithmetic mean of absorbance measured in ELISA tests for each serum dilution was calculated. The sera were collected from the group of immunized animals (5 immunized without adjuvant and 5 immunized with adjuvant) from a control group ( 5 animals immunized only with adjuvant). The levels of $\mathrm{IgG}$ were determined for eight serum dilutions and each individual reaction was repeated three times. Thus, for every serum dilution the level of $\mathrm{IgG}$ was determined based on 15 reactions (5 biological and 3 technical replicates).

\section{Statistical Analyses}

The two-tailed $\mathrm{p}$-value was determined using an unpaired t-test, and $\mathrm{p}<0,05$ was considered significant.

\section{Supporting Information}

Figure S1 Comparison of all putative I. ricinus TROSPA sequences from GB. (DOC)

Materials S1 A - primer sequences. B - DNA sequences encoding OspA protein from B. burgdorferi s. s., B. garinï, and $B$. afzelii.

(DOC)

\section{Author Contributions}

Conceived and designed the experiments: AU MF. Performed the experiments: AU DL CS. Analyzed the data: AU DLJJ-L CS. Contributed reagents/materials/analysis tools: AU MF CS JJ-L. Wrote the paper: AU MF DL.

2. Rar VA, Fomenko NV, Dobrotvorsky AK, Livanova NN, Rudakova SA, et al. (2005) Tickborne pathogen detection, Western Siberia, Russia. Emerg Infect Dis 11: $1708-1715$. 
3. Hamer SA, Roy PL, Hickling GJ, Walker ED, Foster ES, et al. (2007) Zoonotic pathogens in Ixodes scapularis, Michigan. Emerg Infect Dis 13: 1131-1133.

4. Welc-Faleciak R, Hildebrandt A, Sinski E (2010) Co-infection with Borrelia species and other tick-borne pathogens in humans: two cases from Poland. Ann Agric Environ Med 17: 309-313.

5. Capelli G, Ravagnan S, Montarsi F, Ciocchetta S, Cazzin S, et al. (2012) Occurrence and identification of risk areas of Ixodes ricinus-borne pathogens: a cost-effectiveness analysis in north-eastern Italy. Parasit Vectors 5: 61.

6. Paduraru OA, Buffet JP, Cote M, Bonnet S, Moutailler S, et al. (2012) Zoonotic transmission of pathogens by Ixodes ricinus ticks, Romania. Emerg Infect Dis 18: 2089-2090.

7. Chmielewska-Badora J, Moniuszko A, Zukiewicz-Sobczak W, Zwolinski J, Piatek J, et al. (2012) Serological survey in persons occupationally exposed to tick-borne pathogens in cases of co-infections with Borrelia burgdorferi, Anaplasma phagocytophilum, Bartonella spp. and Babesia microti. Ann Agric Environ Med 19: 271-274.

8. Eisen L, Lane RS, editors (2002) Vectors of Borrelia burgdorferi sensu lato. New York: CABI Publishing. 91-115 p

9. Scott JD, Anderson JF, Durden LA (2012) Widespread dispersal of Borrelia burgdorferi-infected ticks collected from songbirds across Canada. J Parasitol 98: 49-59.

10. Hamer SA, Tsao JS, Walker ED, Hickling GJ (2010) Invasion of the Lyme Disease Vector Ixodes scapularis: Implications for Borrelia burgdorferi Endemicity. EcoHealth 7: 47-63.

11. Pal U, Li X, Wang T, Montgomery RR, Ramamoorthi N, et al. (2004) TROSPA, an Ixodes scapularis receptor for Borrelia burgdorferi. Cell 119: 457-468.

12. Konnai S, Yamada S, Imamura S, Nishikado H, Githaka N, et al. (2012) Identification of TROSPA homologue in Ixodes persulcatus Schulze, the specific vector for human Lyme borreliosis in Japan. Ticks Tick Borne Dis 3: 75-77.

13. Abbott A (2006) Lyme disease: uphill struggle. Nature 439: 524-525.

14. Earnhart CG, Buckles EL, Marconi RT (2007) Development of an OspC-based tetravalent, recombinant, chimeric vaccinogen that elicits bactericidal antibody against diverse Lyme disease spirochete strains. Vaccine 25: 466-480.
15. Schuijt TJ, Hovius JW, van der Poll T, van Dam AP, Fikrig E (2011) Lyme borreliosis vaccination: the facts, the challenge, the future. Trends Parasitol 27: $40-47$.

16. Hurkman WJ, Tanaka CK (1986) Solubilization of plant membrane proteins for analysis by two-dimensional gel electrophoresis. Plant Physiol 81: 802-806.

17. Kung F, Anguita J, Pal U (2013) Borrelia burgdorferi and tick proteins supporting pathogen persistence in the vector. Future Microbiol 8: 41-56.

18. Frey A, Di Canzio J, Zurakowski D (1998) A statistically defined endpoint titer determination method for immunoassays. J Immunol Methods 221: 35-41.

19. Lencakova D, Hizo-Teufel C, Petko B, Schulte-Spechtel U, Stanko M, et al. (2006) Prevalence of Borrelia burgdorferi s.l. OspA types in Ixodes ricinus ticks from selected localities in Slovakia and Poland. Int J Med Microbiol 296 Suppl 40: 108-118.

20. Egelkrout E, Rajan V, Howard JA (2012) Overproduction of recombinant proteins in plants. Plant Sci 184: 83-101.

21. Pal U, de Silva AM, Montgomery RR, Fish D, Anguita J, et al. (2000) Attachment of Borrelia burgdorferi within Ixodes scapularis mediated by outer surface protein A. J Clin Invest 106: 561-569.

22. Michel H, Wilske B, Hettche G, Gottner G, Heimerl C, et al. (2004) An ospApolymerase chain reaction/restriction fragment length polymorphism-based method for sensitive detection and reliable differentiation of all European Borrelia burgdorferi sensu lato species and OspA types. Med Microbiol Immunol 193: 219 226.

23. Devriendt B, De Geest BG, Goddeeris BM, Cox E (2012) Crossing the barrier: Targeting epithelial receptors for enhanced oral vaccine delivery. J Control Release 160: 431-439.

24. Sambrook J, editor (2001) chapter 13, protocol 3. 3 ed. New York: Cold Spring Harbor Laboratory.

25. Saluja V, Visser MR, van Roosmalen ML, Leenhouts K, Huckriede A, et al. (2010) Gastro-intestinal delivery of influenza subunit vaccine formulation adjuvanted with Gram-positive enhancer matrix (GEM) particles. Eur J Pharm Biopharm 76: 470-474. 